
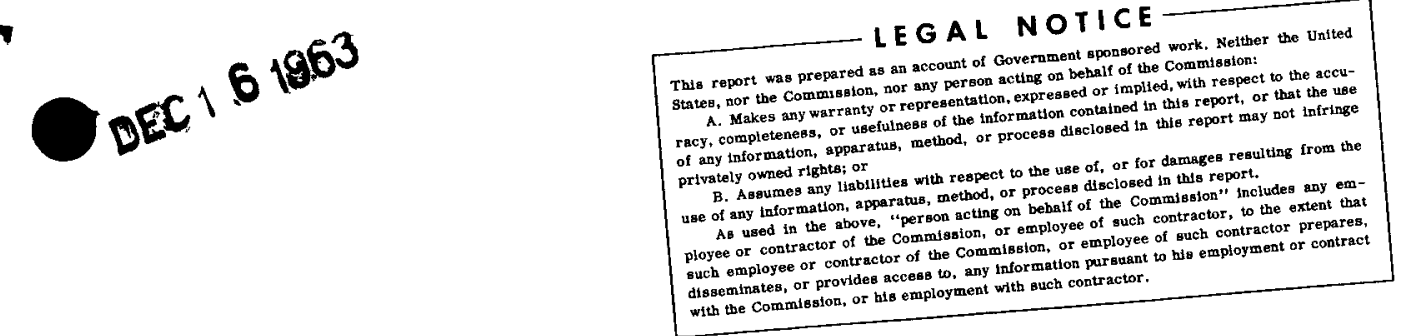

\title{
CHARACTERISTICS OF FUEL COMPOUNDS AND ENCAPSULATION MATERIALS IN \\ RADIOISOTOPE HEAT SOURCES
}

MND-P-2975

April 17, 1963

A report prepared at the request of the Division of Isotopes Development, U. S. Atomic Energy Commission.

Justin L. Bloom

Program Manager

Radioisotope Fuel Technology

NUCLEAR DIVISION

MARTIN COMPANY

BALTIMORE 3, MD.

\begin{abstract}
Facsimile Price $\$$
Microfilm Price $\$$

Available from the Office of Technical Services Department of Commerce Washington 25, D. C.
\end{abstract} , $8 d$ 


\section{DISCLAIMER}

This report was prepared as an account of work sponsored by an agency of the United States Government. Neither the United States Government nor any agency Thereof, nor any of their employees, makes any warranty, express or implied, or assumes any legal liability or responsibility for the accuracy, completeness, or usefulness of any information, apparatus, product, or process disclosed, or represents that its use would not infringe privately owned rights. Reference herein to any specific commercial product, process, or service by trade name, trademark, manufacturer, or otherwise does not necessarily constitute or imply its endorsement, recommendation, or favoring by the United States Government or any agency thereof. The views and opinions of authors expressed herein do not necessarily state or reflect those of the United States Government or any agency thereof. 


\section{DISCLAIMER}

Portions of this document may be illegible in electronic image products. Images are produced from the best available original document. 


\section{INTRODUCTION}

The Division of Isotopes Development, U. S. Atomic Energy Commission, has requested that an evaluation be made of those properties of radioisotope fuel compounds, the materials employed to encapsulate them, and the completed heat source capsules which are important to ensuring the widest utility and safe operation of such sources. For convenience, the properties are grouped into intrinsic and extrinsic categories, i.e., those which are inherent to the materials themselves and those which are the result of some externally applied environmental factor. A third category is employed to cover factors which relate to the interaction - either physical or chemical - of the fuel compound and its encapsulation material. In some instances, the assignment of a property to a category is arbitrary, and any rearrangement which will clarify the problem is solicited.

The present fuel form for strontium-90, strontium titanate, and its encapsulation material, Hastelloy $C$, are analyzed in terms of the significant properties outlined, to determine what remains to be studied to ensure safe operation and to extend operating limits of the fuel-capsule combination.

After the various characteristics have been measured or computed, it should be possible to establish safe operating limits for use of the heat sources in the three broad environments of space, land and sea. 


\section{INTRINSIC PROPERTIES OF RADIOISOTOPE}

\section{FUEL COMPOUNDS}

\section{A. The Radioisotope}

1. Half-life - The half-life must be well-known, since the decay constant derived from it is a factor in determining the specific power, and the thermal power of the isotope decays in accordance with the half-life.

2. Decay scheme - The decay scheme is employed to calculate the specific power of the radioisotope and to compute the amount of shielding required. Spontaneous fission must be considered in the case of alpha emitters.

3. Specific activity - No radioisotope is produced in absolutely pure form. Therefore, dilution of the activity by inert carriers must be known. In some instances, this can be controlled by the isotope production site.

4. Radiochemical impurity concentration - Ordinarily such impurities do not appreciably affect the specific activity by acting as diluents of the desired isotope, but they often cause perturbations in the thermal output-time relationship and may well be the controlling factors in computing shielding. Once again, the concentration of such impurities may be controllable at the production site.

5. Specific power - This factor, usually expressed in watts/kilocurie of isotope or watts/gram of element, may be measured experimentally (the preferable technique) or computed from A1-A4 above. The practical value of watts/gram takes into account the presence of inert diluents. Substantial errors may arise in computing the watts/ kilocurie figure for beta emitters, since the beta energy distribution must be well known.

\section{B. The Chemical Compound or Mixture}

1. Composition - The proportion of radioactive element and its inert analogues in the fuel compound must be known from stoichiometry or should be measured by direct or indirect chemical and radiochemical techniques. If other ingredients are added to perturb the properties of the compound (e. g. , to enhance re-entry burn-up or reduce power density), their effect may vary as a function of concentration, and thus the concentration must be measured or deduced.

2. Density - The theoretical (crystal) density of the material should be known, so that the approach to this figure in processing can be de- 
termined by measurement of the actual density. Normally, maximization of the actual density is desirable, in order to increase the volumetric concentration of the radioisotope.

3. Power density - Expressed in watts/cubic centimeter, the power density is obtained from A5, B1 and B2. Its importance derives from the fact that the capsule designer relies on this figure to determine the fuel volume.

4. Thermal conductivity - This information is required by the capsule designer so that he may compute internal temperatures reached by the fuel as a function of capsule size (and hence of capsule surface temperature), the objective being to keep the fuel temperature below a critical value, such as the melting point. Ideally, the conductivity as a function of temperature is required.

5. Coefficient of expansion - The expansion versus temperature properties of the fuel are desirable for purposes of ensuring that suf ficient volume is available in the capsule at high temperatures. If phase changes occur, this can be particularly troublesome (see below).

6. Melting point - Ideally, the phase diagram for the system is extremely useful, since melting point as a function of composition is then known. The melting point is a factor in establishing the upper limit of operational temperature of the heat source. Recognition must be made of the fact that additives or impurities may alter the melting point of the pure fuel compound. Phase change information is also available from the phase diagram.

7. Mechanical strength - Compressive strength as a function of temperature is a useful figure, since the fuel may be called upon to support the walls of the capsule when the latter is compressed externally. Shear strength and tensile strength data may be required in some applications. Mechanical shock (impact) data would be useful in some instances.

8. Thermal and radiation stability - Decomposition of the fuel as the result of high temperatures and large radiation doses should be evaluated, particularly to determine if gas evolution or crystal growth occurs.

9. Decay effects - The introduction of foreign atoms into the fuel as the result of the decay of the radioactive parent should be evaluated in terms of valence changes and subsequent gas evolution or crystal growth, and of the effect on the other properties noted above.

10. Radiation attenuation - For beta-gamma emitters in particular, two radiation absorption effects are of great significance. The first is beta conversion to heat and bremsstrahlung (plus neutrinos). This process is a function of the average atomic number of the fuel form. For 
heat sources, minimization of high-energy bremsstrahlung formation is highly desirable and is accomplished by reducing the average atomic number - but usually at the expense of a decrease in power density. Since the conversion process is also a function of beta energy distribution, it is extremely difficult to calculate and is best treated experimentally. The other effect is self-absorption of primary gamma radiation or secondary bremsstrahlung, also a function of material density and atomic number. In a massive heat source, increasing the degree of self-absorption of penetrating radiation within the source consequently reduces the amount of external biological shielding required. 
Blank 


\section{EXTRINSIC PROPERTIES OF RADIOISOTOPE}

FUEL COMPOUNDS

1. Gas evolution - Gas may be evolved as part of the primary decay process (e.g., helium from alpha emitters) or by the secondary processes noted earlier.

2. Radiation characteristics - The energy spectrum and dose rate of the radiation in air and as a function of standard absorber thickness must be measured to permit design of adequate shielding.

3. Dissolution or leaching rate - The rate of dissolution of the fuel in naturally occurring fluids should be determined to permit assessment of biological hazards in the event of release of the fuel to the environment.

4. Vapor pressure - In some cases, the vapor pressure of the fuel may be significant at operating temperatures. The capsule designer must have this information to provide assurance that distortion or rupture does not occur.

5. Resistance to thermal shock - In some processing operations or in safety considerations, resistance of the fuel to thermal shock may be a criterion, particularly if its coefficient of expansion is large or phase changes occur.

6. Burnup characteristics - In space applications, it is usually mandatory that the fuel be dispersible into particles one micron or less in diameter upon re-entry from orbital velocities. The effect can be simulated by plasma jet experiments with the fuel form. In some instances, rare at present, re-entry without burnup may be required (i. e. , intact re-entry) and in this event the oxidative or melting effect must be minimized. Some computational evaluation of burnup effects is possible, but heavy reliance upon empirical experiments must be made. 
Blank 
III. INTRINSIC PROPERTIES OF FUEL CAPSULE

1. Physical properties - Ordinarily, the capsule will be fabricated from a metal or alloy, or, in some cases, two different metals or alloys in succession. The usual properties of the materials are required, including tensile and shear strength, creep-rupture data, melting point, coefficient of expansion, thermal conductivity, and density. When these properties are significant functions of temperature, the dependency must be known also. The need for this type of information is ordinarily available from the supplier of the material.

2. Thermal and radiation stability - The capsule material must not undergo phase changes which affect its structure or decompose as the result of increases in temperature up to the operating limit, and it must be able to withstand radiation doses of the order of $10^{12} \mathrm{rad}$ without growth or other deleterious effects. Most structural metals or alloys of the type considered for this application have these attributes. 




Blank 


\section{EXTRINSIC PROPERTIES OF FUEL CAPSULE}

\section{A. Containment Requirements}

1. Bursting strength - If gas evolution is a property of the fuel compound, the capsule must withstand some design internal pressure, and the resulting forces within the walls may be computed based on the physical properties of the capsule material, or - more desirably - the capsule is tested to failure by artificial injection of gas. Long-term creeprupture tests are also of significance and are performed at the maximum anticipated operating temperature.

2. Corrosion rate - An important property of the encapsulation material is its resistance to corrosive attack at elevated temperatures by air, fresh water, and sea water over extended periods of time. This property is measured quantitatively in the conventional units of inches/ year or milligrams/decimeter ${ }^{2}$ day. The type of corrosive attack, e. g. , crevice corrosion or pitting, is needed also. Preliminary screening of capsule materials with no radiation field present is permissible, but final proof testing of a selected material should include measurement of the corrosion rate in the actual radiation environment the capsule will experience. Evaluation of the corrosion rate in terms of such variables as surface finish, temperature of the corrosive medium, presence of organic matter, and differential attack at welded areas is also desirable.

3. Galvanic or electrolytic corrosion - Ordinarily, the capsule will be employed in a system containing other metallic components. The possibility of the establishment of a galvanic cell when the system is immersed in a conducting liquid (e. g. , sea water) cannot be overlooked. Material selection should consider making the capsule or capsules cathodic with respect to the other metals present, so that deposition on, rather than corrosion of the capsule, take place. There is always the possibility that the presence of a radiation field may alter the effect and even reverse it, so this factor should be considered also. If the application is such that a voltage can be applied to the components of the system when it is in a conducting medium, then electrolytic corrosion may take place also.

4. Resistance to thermal shock - Some metals and alloys react unfavorably to large and sudden changes in temperature, particularly if they undergo phase changes. For example, it is conceivable that a hot capsule could be immersed suddenly in liquid oxygen if it is a device aboard a missile and a launch abort occurs. If mechanical strength is a requirement for that particular capsule, it may suffer a deleterious effect by such treatment. 
5. Resistance to impact - Under conditions incident to ground trans portation accidents, capsules may be subjected to impact forces of the order of $10 \mathrm{~g}$ in almost any orientation. If involved in aircraft accidents, terminal velocity impacts of the order of $300 \mathrm{fps}$ could be experienced by the capsules. Impacts resulting from malfunction of missile boosters would be of the latter order of magnitude also. Depending on the utilization of the capsules, any or all of these conditions might be anticipated and the capsules would have to maintain their integrity without release of their radioactive contents. Actual impact testing to accumulate empirical data is the only reliable way to ensure capsule integrity for this type of imposed condition. For some special applications, high-velocity accidental impacts on other celestial bodies may determine the design of the capsule to withstand or partially withstand rupture. Velocities up to $10,000 \mathrm{fps}$ have been considered.

6. External pressure - Two types of accident environment may subject the capsules to high external pressures which could result in collapse and subsequent release of radioactivity. The first results from explosion of a missile booster, in which case a shock overpressure is followed by a decaying static pressure. The other results from accidental immersion of a capsule in deep water, where uniform hydrostatic pressures are experienced. Temperature conditions are radically different in the two cases and must be taken into account. Once again, empirical testing is the reliable method of evaluating the quality of capsules under these conditions.

7. Fire and explosion - It has been implied already that capsules must withstand unusually high transients of temperature and pressure in the event of involvement in a transportation accident or missile abort situation. Standard test conditions for transportation accidents are being evolved by several Government agencies.

\section{B. Burnup Characteristics}

1. Aerodynamic heating - Capsules intended for space applications may undergo aerodynamic heating, either as the result of re-entry to the atmosphere because of missile malfunction, or because decay of an orbital trajectory results in re-entry. Comparable heating rates can be simulated by plasma jet tests, but the effect of continuing changes in atmospheric density and composition must be treated analytically in most instances. Two opposing criteria may be required relative to capsule integrity under re-entry conditions: intact re-entry is usually required for the missile abort condition, whereas complete oxidation of the container and the dispersion of its contents at high altitude are required for re-entry from orbit. Furthermore, in some special applications, intact re-entry from orbital or super-orbital velocities may be mandatory and recourse to the use of protective ablative materials around the capsule may be required to meet this criterion. 
C. Miscellaneous

1. Fabricability - Included under this general term are such properties of the metal or alloy as its ability to be machined with conventional tools, its availability in useful shapes, and its ability to be welded to the requisite degree of penetration without the use of brazes or other alloys which do not exhibit the same properties as the base metal. A further restriction placed on the welding criterion is that it is usually necessary to perform at least one weld on a capsule in a dry box or hot cell by remote or semiremote means.

2. Radiation attenuation - The capsule contributes some measure of shielding to the fuel, depending on its wall thickness relative to the amount of fuel. Therefore, $t_{1}$ s characteristics of the encapsulation material for the attenuation of penetrating radiation should be reasonably well known. 
Blank 
V. COMPATIBILITY OF FUEL AND CAPSULE

1. Chemical compatibility - Quite obviously, chemical reaction of the fuel and capsule material cannot be permitted to occur, primarily because of potential deleterious effects on the capsule. Since the two materials are ordinarily at high temperature, solid state reactions are conceivable, as are alloying and diffusion. The presence of a radiation field may either enhance or inhibit reaction. If necessary, an inner capsule, to which no structural attributes are assigned, but which acts as a corrosion barrier, can be employed. With radioactive materials, the fact that new chemical species are produced by decay of the parent nuclei must be considered. Due to valence changes on decay, oxygen may be liberated from oxide fuels, and it can react with the encapsulation material.

2. Physical compatibility - Sufficient clearance and void volume must be allowed in the capsule design to account for growth of the fuel by processes already described, gas evolution and consequent internal pressure increase, and differential coefficients of expansion. 
-

Blank

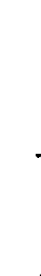




\section{EVALUATION OF STRONTIUM TITANATE FUEL AND HASTELLOY C ENCAPSULATION MATERIAL IN TERMS OF OUTLINED CHARACTERISTICS}

Based on the previous sections, it is possible to make an evaluation of the present status of the strontium titanate fuel form for strontium90 , and for the Hastelloy $C$ capsules presently used to contain it. The paragraph headings below refer to corresponding paragraphs in the previous sections.

\section{INTRINSIC PROPERTIES OF RADIOISOTOPE F UEL COMPOUNDS}

A. The Radioisotope (Sr-Y-90)

1. Half-life - 27. 7 years, probably accurate to \pm 0.3 year or $\pm 1 \%$. This value is sufficiently good for all practical purposes.

2. Decay scheme - The maximum beta energies for $\mathrm{Sr}-90$ (0.54 Mev) and $\mathrm{Y}-90$ (2. $26 \mathrm{Mev)} \mathrm{reported} \mathrm{in} \mathrm{the} \mathrm{literature} \mathrm{are} \mathrm{accurate}$ enough for practical purposes. However, the beta energy distributions for these isotopes have not been measured, to our knowledge, and the calculated distributions vary substantially from worker to worker.

3. Specific activity - The theoretical specific activity of 143 curies/ gram $\mathrm{Sr}-90$ (based on the half-life given above) is reduced by the presence of stable strontium isotopes to a present maximum of 80.4 curies/ gram total $\mathrm{Sr}$, since the $\mathrm{Sr}-90$ proportion of total $\mathrm{Sr}$ is not more than $56 \%$ with current reactor operating conditions. This value is further reduced by dilution of the other principal alkaline earth cations, $\mathrm{Ca}$ and $\mathrm{Ba}$. The latter effect is a variable controllable by the production site; $\mathrm{Ca}$ and $\mathrm{Ba}$ have comprised from 1 to $20 \%$ by weight of the total cations. Thus the practical specific activity of $\mathrm{Sr}-90$ ranges from 79.5 to 64.3 curies/gram of total cations.

4. Radiochemical impurity concentration - $\mathrm{Sr}-89$ and $\mathrm{Ce}-144$ are the principal radiochemical impurities present in $\mathrm{Sr}-90$. The $\mathrm{Sr}-89$ $(\mathrm{T}-1 / 2=51$ days $)$ is present in considerable quantity in fresh fission products, and since it cannot be removed chemically, it must be allowed to decay to a reasonable level before the $\mathrm{Sr}-90$ is purified and shipped. Since 3.4 watts/kilocurie of thermal power are produced by $\mathrm{Sr}-89$, it will perturb the operation of systems using the thermal power of Sr-90. The present specification calls for less than 0.11 curie Sr89 per curie $\mathrm{Sr}-90$ in the completed heat source, meaning that more than a year of preliminary decay is required. The Ce-144 $(\mathrm{T}-1 / 2=$ 285 days) is a significant primary gamma emitter because its shortlived daughter emits $2.18 \mathrm{Mev}$ gammas in $1 \%$ of its decay. This high energy radiation can be the controlling factor in the amount of shielding required for a heat source. 
5. Specific power - The actual specific power of Sr-Y-90 is somewhat uncertain for the reasons noted in (2) on the preceding page. ORNL has reported informally that the value is 6.3 watts/kilocurie; J. Silverman has reported a value of 6.0 watts/kilocurie. Our experimental work at Quehanna indicates that the value is 6.3 watts/kilocurie by an indirect method. An accurate calorimetric determination of this important value is required.

B. The Chemical Compound or Mixture $\left(\mathrm{SrTiO}_{3}\right)$

1. Composition - No direct method of analyzing the radioactive fuel form for the proportions of $\mathrm{Sr}, \mathrm{Ti}$, and $\mathrm{O}$ exists at this time. Proportions are computed from materials balance figures, the thermal output of $\mathrm{Sr}-90$, and the weight ratio of cations in the feed material.

2. Density - A theoretical density of 5.11 grams/cubic centimeter has been measured for $\mathrm{SrTiO}_{3}$ by NBS, using X-ray diffraction techniques. This value is perturbed by the presence of $\mathrm{CaTiO}_{3}$ and $\mathrm{BaTiO}_{3}$ in the practical compound. Experimental determination of the perturbation has not been made, but it can be computed with reasonable accuracy by the method of mixtures. The actual density is a function of empirical processing conditions and may vary somewhat if exact stoichiometry is not maintained. Actual densities of 3.0 to 4.6 grams/cubic centimeter in pellet form have been measured. This factor is important as a process variable and can be measured empirically.

3. Power density - Because of the inherent variations in density and in impurity concentrations that are possible, the power density of $\mathrm{SrTiO}_{3}$ has varied from 0.6 to 0.9 watt/cubic centimeter. The maximum value, assuming $100 \%$ compaction, exact stoichiometry, and no stable impurities other than stable strontium isotopes, is 1.24 watts/ cubic centimeter, if the specific power of $\mathrm{Sr}-90$ is taken to be 6.3 watts/ kilocurie.

4. Thermal conductivity - The conductivity of the titanate has been measured near room temperature and has been found - as expected to be a function of density. At $88.8 \%$ of theoretical density, the value is $0.0132 \mathrm{cal} / \mathrm{sec}^{-\mathrm{cm}^{-}}{ }^{\circ} \mathrm{C}$, and at $94 \%, 0.0173$. Although the values will be different at the operating temperatures of about $1200^{\circ} \mathrm{F}$ employed, they are sufficiently good for design purposes at present. The difficulty of measuring conductivities at high temperature is severe, and it is probably not worthwhile to attempt to refine these figures.

5. Coefficient of expansion - The value has been measured to be $11.2 \times 10^{-6} \mathrm{in.} / \mathrm{in} .^{\circ} \mathrm{C}$ over the range $100^{\circ}$ to $700^{\circ} \mathrm{C}$. This value is sufficiently accurate for design purposes. 
6. Melting point $-\mathrm{R}$. Roy reports a value of $1910^{\circ} \mathrm{C}$ for the melting point. However, the phase diagram for the system $\mathrm{SrO}-\mathrm{TiO}_{2}$ prepared by him indicates that the melting point changes sharply as deviation from exact stoichiometry occurs. At present, this has posed no problem since the operational temperatures employed have not approached the melting point within $1000^{\circ} \mathrm{C}$. Melting points lower than $1440^{\circ} \mathrm{C}$ should not be experienced in practice, since this value represents the eutectic of $\mathrm{SrTiO}_{3}$ and $\mathrm{TiO}_{2}$; in practice, attempts are made to keep the mixture slightly rich in $\mathrm{TiO}_{2}$. No precise information is available on solidstate phase changes in the $\mathrm{SrO}-\mathrm{TiO}_{2}$ system.

7. Mechanical strength - No information is available on the strength properties of $\mathrm{SrTiO}_{3}$. It can be inferred, however, that the material is weak in bending and in shear, but can withstand compressive loads of the order of $10,000 \mathrm{psi}$ in the sintered pellet form.

8. Thermal and radiation stability - No information is available on this subject. By inference, the material is probably stable thermally at anticipated operating temperatures, but its radiation stability is open to question. The effect of radiation is extremely important and should be evaluated.

9. Decay effects - Introduction of $\mathrm{Zr}$ into the lattice by decay may cause crystal changes. However, if no valence change occurs, the lattice may accommodate the $\mathrm{Zr}$ without difficulty. This effect is important, and should be evaluated.

10. Radiation attenuation - No experimental measure of the selfabsorption of beta or gamma (including bremsstrahlung) radiation is available to us. Spamer has computed gamma absorption coefficients for $\mathrm{SrTiO}_{3}$ to be as follows:

\begin{tabular}{ll}
$\mu_{\mathrm{O}}, \mathrm{cm}^{-1}$ & E, Mev \\
\hline 2.11 & 0.1 \\
0.98 & 0.15 \\
0.68 & 0.2 \\
0.48 & 0.3 \\
0.41 & 0.4 \\
0.36 & 0.5 \\
0.33 & 0.6
\end{tabular}


$\underline{\mu_{0}, \mathrm{~cm}^{-1}}$

0.29

0.287

0.21

0.18

0.15 $\underline{\text { E, Mev }}$

0.8

1.0

1.5

2.0

3.0

Experimental confirmation of these values would be useful, since build-up is not taken into account in the calculations.

Because of the high density of $\mathrm{SrTiO}_{3}$, only a small fraction of the high energy beta particles will leave the source. The actual range of betas as a function of beta energy would be of extreme interest for fabrication of beta sources, but is not of particular importance in heat source design.

\section{EXTRINSIC PROPERTIES OF RADIOISOTOPE}

\section{FUEL COMPOUNDS}

1. Gas evolution - No gas evolution is anticipated from $\mathrm{SrTiO}_{3}$, but the effects of radiation damage are unknown and hence there is the remote possibility that oxygen could be evolved. Storage of a radioactive pellet in a controlled atmosphere could prove or disprove this point.

2. Radiation characteristics - Direct measurements of either dose rate or energy spectrum from radioactive $\mathrm{SrTiO}_{3}$ have not been made. This information would be very valuable for use in shielding design.

3. Dissolution or leaching rate - Tracer studies of the rate of dissolution of $\mathrm{SrTiO}_{3}$ tagged with $\mathrm{Sr}-85$ or $\mathrm{Sr}-90$ have been made by NRDL and Martin. The results were in good agreement, indicating that the rate is of the order of $1 \mathrm{microgram} / \mathrm{cm}^{2}$ day in sea water. Equivalent values were found by NRDL in fresh water. However, the dissolution rate of the fully radioactive titanate remains open to question and should be determined.

4. Vapor pressure - $\mathrm{SrTiO}_{3}$ is employed at temperatures so far below its melting point or that of its constituents that vapor pressure should be of no consideration. 
5. Resistance to thermal shock - This property has not been evaluated for the titanate.

6. Burnup characteristics - This property, a highly important one for space applications, has not been studied at the time of preparation of this document.

\section{INTRINSIC PROPERTIES OF FUEL CAPSULE}

\section{(HASTELLOY C)}

1. Physical properties - The following data are available from the manufacturer:

a. Corrosion penetration rate in common reagents at room temperature, $150^{\circ} \mathrm{F}$, and boiling point of the reagent.

b. Chemical composition.

c. Density.

d. Melting point.

e. Electrical resistivity.

f. Mean coefficient of thermal expansion as a function of temperature.

g. Thermal conductivity as a function of temperature.

h. Specific heat.

i. Magnetic permeability.

j. Casting shrinkage.

k. Room temperature and hot hardness.

1. Formability.

m. Impact strength (Izod v-notch).

n. Short-time tensile data as functions of temperature.

o. Stress-rupture data as functions of temperature.

p. Creep data as functions of temperature. 
2. Thermal and radiation stability - Thermal stability of Hastelloy C should be excellent at all temperatures approaching its melting point, as long as it is in the unstressed condition. No data are available on the radiation stability, but as a metallic alloy, Hastelloy $\mathrm{C}$ should not suffer in this respect.

\section{EXTRINSIC PROPERTIES OF FUEL CAPSULE}

(HASTELLOY C)

A. Containment Requirements

1. Bursting strength - No tests have been performed on Hastelloy $C$ capsules. This information is not required at the present time, and will become important only if gas evolution from the fuel can be demonstrated.

2. Corrosion rate - Since Hastelloy $\mathrm{C}$ finds use in industry principally because of its corrosion resistance, a considerable amount of information on this property is available. However, none of it is directly applicable to radioisotope heat sources, since no realistic measurements have been made in radiation fields of the type that would exist from $\mathrm{SrTiO}_{3}$. Such information is highly desirable, since one cannot predict easily whether radiation will enhance or inhibit the corrosion rate as measured under conventional conditions.

3. Galvanic or electrolytic corrosion - Once again, no substantial information is available on the effect of radiation on electrolytic cells established between two pieces of Hastelloy $\mathrm{C}$ or between Hastelloy $\mathrm{C}$ and other metals. Some experimental data collected at Martin indicate that with an electrolytic cell of Hastelloy $\mathrm{C}$ anode and cathode in sea water and an induced current of two amperes, the anode was badly corroded and the cathode was unchanged. With a platinum anode and Hastelloy $\mathrm{C}$ cathode, neither electrode showed any detectable weight change or apparent damage. No information on galvanic corrosion is known to us.

4. Resistance to thermal shock - The manufacturer states, "Hastelloy $\mathrm{C}$ is particularly useful where parts are either highly stressed or subject to repeated thermal shock at temperatures from $1600^{\circ}$ to $1800^{\circ} \mathrm{F} .^{\prime \prime}$ The conditions of test might have been less stringent than those that could be applied for a space fuel capsule, but appear to be satisfactory for terrestrial applications.

5. Resistance to impact - Hastelloy $\mathrm{C}$ has not been evaluated in capsule geometries and under impact conditions relating to space applications or aircraft accidents. Capsules now in use could almost certainly survive nominal drop tests of the type encountered in ground 
transportation accidents. However, the actual tests have not been performed.

6. External pressure - Two SNAP 7E capsules, one loaded with simulated fuel and the other empty, have been subjected to hydrostatic pressures up to $10,000 \mathrm{psi}$ without failure. The failure point is unknown. The larger SNAP 7B-7D capsules have not been tested.

7. Fire and explosion - No test data are available on the resistance of Hastelloy $\mathrm{C}$ capsules to fire and explosion. Tests with comparable materials (Inconel $\mathrm{X}$, Haynes 25) indicate that Hastelloy $\mathrm{C}$ would survive such conditions when fabricated in the SNAP 7 configuration.

B. Burnup Characteristics

1. Aerodynamic heating - No test data are available on the burnup properties of Hastelloy C.

C. Miscellaneous

1. Fabricability - Hastelloy $\mathrm{C}$ is more difficult to machine and form than stainless steel. However, much experience exists in industry on the fabrication of it into complex shapes. It is readily available in sheet, wire, rod, bar, plate and tubing. It can be fusion or filler welded (using Hastelloy $C$ rod) with excellent results. No real problem exists in this area, except that no method is known for nondestructively measuring weld penetration.

2. Radiation attenuation - Gamma radiation absorption coefficients for Hastelloy $\mathrm{C}$ have been computed by Spamer, as follows:

\begin{tabular}{cc}
$\mu_{\mathrm{o}}, \mathrm{cm}^{-1}$ & E, Mev \\
\hline 6.22 & 0.1 \\
2.63 & 0.15 \\
1.68 & 0.2 \\
1.09 & 0.3 \\
0.885 & 0.4 \\
0.777 & 0.5 \\
0.700 & 0.6 \\
0.603 & 0.8 \\
0.538 & 1.0
\end{tabular}




$$
\mu_{\mathrm{o}}, \mathrm{cm}^{-1}
$$

0.427

0.384

0.329
$\underline{E, M e v}$

1.5

2

3

Experimental verification of these numbers would be desirable.

\section{COMPATIBILITY OF FUEL AND CAPSULE}

1. Chemical compatibility - No tests of the compatibility of radioactive $\mathrm{SrTiO}_{3}$ and Hastelloy $\mathrm{C}$ have been made, but closely analogous experiments have been conducted in which the chromium and cobalt cermets of $\mathrm{SrTiO}_{3}$ were heated in an argon atmosphere on plates of Hastelloy $\mathrm{C}$, for 120 hours at $800^{\circ} \mathrm{C}$. No reaction was observed. This effect is probably not critical up to temperatures approaching the melting point of Hastelloy C.

2. Physical compatibility - Enough data exist on thermal expansion properties to ensure that no mismatch of dimensions could occur. No appreciable void volume for gas pressure buildup has been employed in SNAP 7 capsules, since the probability of oxygen evolution is so low. 